\title{
La Tomografía por Emisión de Positrones (PET) en la práctica clínica oncológica
}

\author{
J. P. Suárez Fernández*, A. Maldonado Suárez*, M. L. Domínguez Grande**, J. A. Serna Macías****, \\ O. Kostvinseva*, A. Ordovás Oromendía*, E. Castell*, C. Martín*, E. Gorospe*, J. M. Alfonso Alfonso*
}

\section{Resumen}

La Tomografía por Emisión de Positrones con 18F-fluorodeoxiglucosa (PET-FDG) es una técnica de diagnóstico por imagen cuyo uso se ha generalizado en España durante la última década. Existen una serie de indicaciones concretas, en las cuales la PET-FDG ha demostrado sobradamente su superioridad con respecto a los métodos convencionales de diagnóstico. Es recomendable, por ello, realizar un estudio PET-FDG únicamente en las siguientes situaciones: la estadificación de tumores pulmonares y linfomas, la re-estadificación de tumores de tiroides, colorrectales, de cabeza-cuello, linfomas y melanomas; la localización de tumores de origen desconocido; la caracterización del nódulo pulmonar solitario; $y$, por último, el diagnóstico diferencial recidiva/radionecrosis en tumores cerebrales.

Palabras clave:

Tomografía por Emisión de Positrones (PET). 18FDG. Oncología.

Oncología, 2004; 27 (8):479-489

* Centro PET Complutense (Madrid)

** Servicio de Medicina Nuclear. Hospital Universitario Central de Asturias (Oviedo)

*** Hospital Angeles del Pedregal. México D.F. (México) 


\section{J. P. Suárez Fernández y cols.}

\section{Summary}

Positron emission tomography with 18F-fluorodeoxyglucose (FDG-PET) is a diagnostic procedure which has been progressively incorporated to clinical practice in Spain during the last decade. There are several indications in which FDG-PET has demonstrated a better diagnostic accuracy when compared with conventional methods. In order to optimize the use of this new technique, it is recommendable to select those situationts where FDG-PET has shown to be clearly better than the conventional techniques, as staging of lung cancer and lymphoma; restaging of thyroid cancer, colorectal cancer, head and neck cancer, lymphoma and melanoma; localization of unknown primary site carcinomas; diagnosis of solitary pulmonary nodules; and differentiation of tumor recurrence from radionecrosis in brain tumors.

Key words: Positron emission tomography. PET. FDG-PET. 5FUDG. Oncology.

\section{Introducción}

La tomografía por emisión de positrones (PET) es una técnica de Medicina Nuclear que permite obtener imágenes de la distribución in vivo de diferentes moléculas. Es lo que se ha denominado la "imagen molecular", complemento de la información anatómica que proporcionan la tomografía axial computerizada (TAC) o la resonancia magnética (RM). Esto se consigue mediante la administración intravenosa de un radiofármaco, que es la unión de un isótopo radioactivo con una molécula determinada, tras lo cual se realiza la adquisición de las imágenes en una cámara PET.

En la práctica clínica diaria, la molécula que se utiliza es la fluorodesoxiglucosa (FDG) que va unida al ${ }^{18} \mathrm{~F}$, isótopo radioactivo emisor de positrones de período de semidesintegración muy corto (110 minutos). La FDG es un análogo de la glucosa y es captada tanto por las células normales como por las cancerígenas, pero no sigue el ciclo bioquímico normal de la glucosa, por lo que queda atrapado en su interior. De forma fisiológica, algunos tejidos como el cerebro, el miocardio o el hígado tienen una alta demanda de glucosa, lo que se va a traducir en una alta retención de FDG (Fig. 1). En el caso de las células malignas, su alta tasa metabólica implica igualmente una gran avidez por la FDG, cuyo paso al interior celular está además muy potenciado, lo que permite a priori diferenciar los tejidos malignos de los benignos.

No obstante, la imagen obtenida tiene limitaciones en cuanto a la detección de la enfermedad de muy bajo grado de malignidad, debido precisamente a una menor avidez por la glucosa; del mismo modo, también puede mostrar zonas de elevada actividad celular de causa benigna, como la inflamación o la infección, que serán causas potenciales de falsos positivos. Ade-

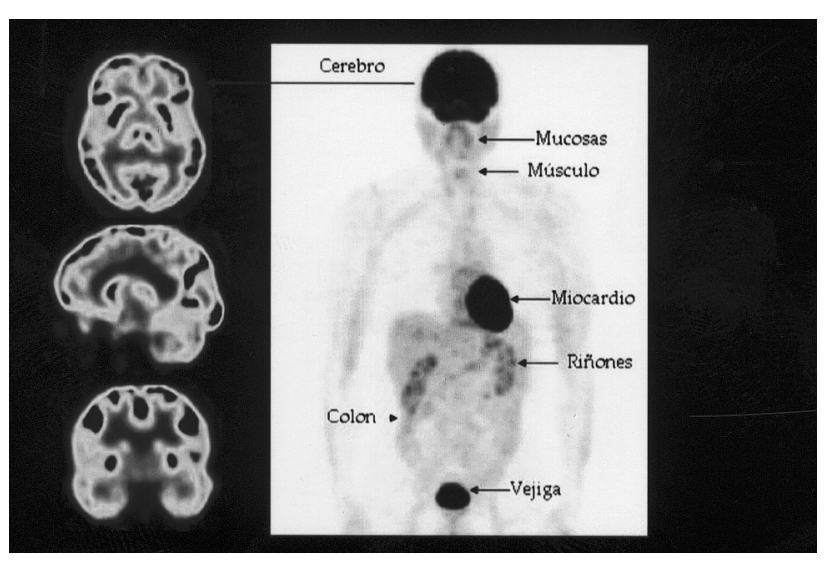

Fig. 1. Estudio PET-FDG normal.

más, aunque las cámaras PET poseen una aceptable resolución espacial $(4-5 \mathrm{~mm})$, cabe señalar que la imagen obtenida no será capaz de detectar la enfermedad microscópica.

La PET es una técnica de imagen que surge en la década de los 70 en Estados Unidos, implantándose en la práctica clínica diaria durante la década de los 90. La PET fue introducida en España en 1995 (Centro PET Complutense, Madrid). En la actualidad hay cerca de 30 centros PET distribuidos por toda la geografía española. Desde el año 2000, los hospitales públicos de las diferentes comunidades autónomas están incorporando esta tecnología a la batería de pruebas diagnósticas existentes. La ${ }^{18} \mathrm{~F}-\mathrm{FDG}$ es el radiofármaco que ha permitido la introducción de la técnica PET a nivel hospitalario, a pesar de que existen en la actualidad cerca de 3.000 radiofármacos sintetizados; de aquí en adelante, se sobreentenderá al referirnos a la PET como a aquella realizada con ${ }^{18} \mathrm{~F}-\mathrm{FDG}$ (PET-FDG). 
TABLA I

\section{Indicaciones de uso tutelado de la PET (Ministerio de Sanidad y Consumo)}

\section{Situación clínica}

Melanoma maligno recurrente susceptible de cirugía radical Carcinoma colorrectal recurrente potencialmente operable Nódulo pulmonar solitario radiológicamente indeterminado Carcinoma de pulmón no microcítico

Tumores cerebrales ya tratados

Linfomas Hodgkin y No-Hodgkin

Tumores malignos de cabeza y cuello recidivantes susceptibles de cirugía radical

Cáncer de tiroidesconocido
Indicación

Re-estadificación

Re-estadificación

Diagnóstico benignidad / malignidad

Estadificación preterapéutica.

Diagnóstico diferencial recidiva/radionecrosis

Estadificación, re-estadificación y valoración de masa residual

Re-estadificación

Localización del tumor primario
La Oncología es la principal indicación clínica de la PET-FDG (más del $90 \%$ de las indicaciones). Debido a su capacidad de poder visualizar lesiones malignas de moderado-alto grado, la PET-FDG es de gran utilidad en las neoplasias malignas de mayor incidencia en la población, tales como las de pulmón, mama, colon, linfomas, cabeza y cuello o melanoma. Dado que estamos analizando el metabolismo de las células cancerígenas, su detección será más precoz que las alteraciones morfológicas, parámetro utilizado por la TAC o la RM. Además, se podrán evaluar también de forma más precoz los efectos de los tratamientos tanto quimio como radioterápicos, en comparación con esas técnicas convencionales. La falta de definición anatómica de la PET está siendo paliada con los nuevos equipos híbridos PET-TAC. Estosn proporcionan al especialista una visión completa de la enfermedad, especialmente cuando se planifiquen procedimientos quirúrgicos.

El Ministerio de Sanidad y Consumo ha sometido a Uso Tutelado 9 indicaciones PET-FDG en Oncología desde 2002, que detallaremos a continuación.

\section{Indicaciones clínicas de la PET en Oncología}

En España se autoriza la realización de estudios PET-FDG en determinadas situaciones clínicas (Tabla I), recogidas en el Protocolo de Uso Tutelado de mayo de $2002^{1,2}$, como paso previo a su inclusión en las prestaciones del Sistema Nacional de Salud.

\section{Tumores del sistema nervioso central}

En el tratamiento de los tumores del sistema nervioso central se han introducido técnicas tales como la radioterapia intersticial, la braquiterapia y la radiocirugía estereotáxica, que permiten una mayor supervivencia de los pacientes. Como contrapartida, existe una mayor incidencia de daño cerebral post-radioterapia, que se suele producir en un $50 \%$ de los casos tras radiocirugía y en un $65 \%$ tras radioterapia intersticial. Transcurrido un cierto período de tiempo, el paciente puede experimentar nuevamente síntomas que dependen de las secuelas de la terapia o bien de una recidiva, siendo en muchas ocasiones difícil diferenciar una causa de otra, lo que dificulta la conducta terapéutica que se debe seguir. Las técnicas de imagen anatómicas o funcionales (TAC, RM, SPECT) son poco específicas en dicho diagnóstico diferencial. La sensibilidad de la PET-FDG en esta situación clínica varía, según los trabajos y protocolos empleados, del 73 al 100\%. La especificidad del PET-FDG es del 56 al $100 \%$ y los valores predictivos positivos y negativos son 80 y $46 \%$, respectivamente. Comparada con la RM la PET resulta ser superior, tanto en la detección de recidiva/restos tumorales como en la encefalopatía post-tratamiento ${ }^{3-}$ 7. El máximo rendimiento se obtiene con la imagen de fusión PET-RM.

Actualmente, el criterio de indicación de la PET-FDG en los tumores del SNC es ${ }^{1,2}$ :

- Pacientes con gliomas grados I y II que presenten síntomas compresivos y/o irritativos, con RM con contraste que no discrimine entre recidiva tumoral y secuelas post-tratamiento (radionecrosis o cicatrices).

\section{Cáncer de cabeza y cuello}

La supervivencia del tumor de cabeza y cuello (60\%) no ha variado desde el advenimiento de la terapia 


\section{J. P. Suárez Fernández y cols.}

combinada (cirugía+radioterapia) a causa de las metástasis y de los segundos tumores primarios. Detectar rápidamente las recurrencias bajaría la mortalidad. La PET-FDG es más segura para el diagnóstico diferencial entre recurrencia tumoral y cambios fibróticos $y / 0$ necróticos post-tratamiento que la TAC $\circ \mathrm{RM}$, teniendo un importante papel en la detección precoz de dichas recurrencias y en la detección de segundos tumores primarios. La PET-FDG puede resultar también útil para determinar el lugar más adecuado para realizar la biopsia. En ocasiones, nos encontramos ante metástasis ganglionares cervicales de tumor de origen desconocido a pesar de un completo estudio de extensión. En estos casos la PET-FDG presenta una capacidad diagnóstica superior a otras técnicas. Por último, en la monitorización del tratamiento la PET-FDG muestra una mayor especificidad que las técnicas morfoestructurales, diferenciando los pacientes que responden al tratamiento y los que no responden, en los que permitirá modificar la pauta terapéutica de forma precoz ${ }^{8-17}$.

Actualmente, el criterio de indicación de la PET en el cáncer de cabeza y cuello es ${ }^{1,2}$ :

- Pacientes con sospecha de recurrencia, tras la realización de todas las pruebas diagnósticas convencionales, susceptibles de cirugía radical.

\section{Cáncer de tiroides}

Una mayor capacidad diagnóstica de la PET-FDG frente a las demás técnicas convencionales es el diagnóstico de recurrencia locorregional, ganglionar regional y a distancia del carcinoma diferenciado de tiroides cuando el rastreo con ${ }^{131} \mid$ es negativo, y resulta de mayor valor cuando las cifras de Tiroglobulina (Tg) se encuentran elevadas y cuando el paciente está bajo terapia sustitutiva. La PET-FDG resulta superior en la detección de recurrencias ganglionares cervicales a las demás técnicas de imagen que basan su diagnóstico en el tamaño de la lesión. La PET-FDG ha demostrado la existencia de metástasis en ganglios de tamaño normal y en un $45 \%$ de pacientes sin elevación de Tg. Los resultados de la PET-FDG en estos pacientes implican cambios en el manejo terapéutico de los mismos, llevándoles en determinados casos a cirugías con intención curativa ${ }^{18-24}$. Por otro lado, el hecho de realizar la PET-FDG con estimulación, preferentemente exógena de la TSH, puede producir un incremento de su rendimiento diagnóstico. También es de gran utilidad en el carcinoma medular ante elevación progresiva de la calcitonina en sangre.

Actualmente, los criterios de indicación de la PET en el cáncer de tiroides son ${ }^{1,2}$ :

- Pacientes en seguimiento tras un diagnóstico y tra- tamiento iniciales de un cáncer diferenciado de tiroides con sospecha de recurrencia o metástasis susceptibles de tratamiento (aumento de la tiroglobulina con rastreos con ${ }^{131}$ | negativos), tras la realización de todas las pruebas diagnósticas convencionales.

- Pacientes diagnosticados y tratados previamente de un carcinoma medular de tiroides con calcitonina alta y pruebas de imagen negativas o indeterminadas con el objeto de identificar restos de tejido tiroideo susceptibles de ser resecados quirúrgicamente.

\section{Cáncer de pulmón}

El carcinoma broncogénico representa el $95 \%$ de los tumores primarios pulmonares, siendo la primera causa de mortalidad por cáncer. El $75 \%$ de ellos son carcinomas no microcíticos, en los que es posible, al ser localizados, realizar una resección potencialmente curativa. La detección en los ganglios linfáticos de la afectación carcinomatosa es problemática con el TAC, ya que el resultado de este método parece normal en algunos casos en que existe afectación metastásica de los mismos y anormal en ganglios no malignos que simplemente han aumentado de tamaño. En el diagnóstico de tumor primario de $>2 \mathrm{~cm}$, la PET-FDG presenta igual sensibilidad que la SPECT con ${ }^{201} \mathrm{Tl}$, $99 \mathrm{mTC}$ MIBI o ${ }^{99 \mathrm{~m} T c-T e t r o f o s m i ́ n, ~ p e r o ~ p a r a ~ l e s i o n e s ~ d e ~ m e n o r ~}$ tamaño es claramente superior la PET-FDG. La PET es superior a la TAC y al SPECT con MIBI o Tetrofosmín en la detección de ganglios mediastínicos afectados por el cáncer de pulmón y mejora la estadificación $\mathrm{N}$ cuando se asocian PET y TAC. El alto valor predictivo de esta técnica permite evitar procedimientos agresivos diagnósticos como la mediastinoscopia, reduciendo, por tanto, los costes diagnósticos y evitándole así al paciente la morbilidad asociada a los mismos (Fig. 2). La PET-FDG detecta metástasis extratorácicas no sospechadas por otras técnicas, lo que supone un cambio en la estadificación tumoral en un considerable porcentaje de pacientes, descartando en ellos la resección quirúrgica como tratamiento curativo y evitando cirugías innecesarias. En la detección de metástasis óseas, la PET-FDG presenta la ventaja añadida de ser más específica y presentar un mayor valor predictivo positivo que la gammagrafía ósea. También resulta más adecuada que la TAC para predecir la respuesta a la quimioterapia de inducción tanto del tumor como a nivel ganglionar, por lo que se puede considerar como buena técnica para seleccionar los pacientes que se beneficiarán de un tratamiento locorregional con intención curativa ${ }^{25-35}$.

Actualmente, los criterios de indicación de la PET en el cáncer de pulmón son ${ }^{1,2}$ : 


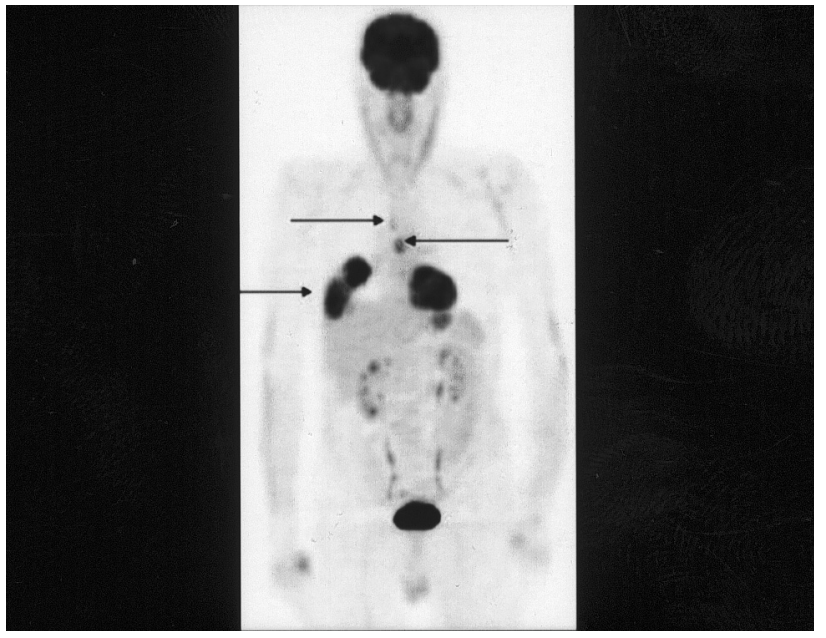

Fig. 2. Estudio de estadificación de cáncer de pulmón no microcítico. Masa tumoral maligna en pulmón derecho, con extensión a pared torácica y presencia de adenopatías mediastínicas.

- Pacientes con cáncer de pulmón no microcítico, tras la realización de todas las pruebas diagnósticas convencionales de estadificación, con el fin de establecer la extensión y tasa de progresión de la enfermedad para realizar cirugía radical.

- No se realizará PET con la indicación de re-estadificación.

\section{Nódulo pulmonar solitario}

Un nódulo pulmonar solitario (NPS) es una lesión única esférica dentro del pulmón que no está asociada a agrandamiento hiliar o atelectasia y cuyo tamaño es generalmente menor de $4 \mathrm{~cm}$ de diámetro. Cuando son malignos representan aproximadamente el 15\% de los tumores malignos pulmonares. La mitad de los NPS suelen ser benignos (el $80 \%$ de ellos son granulomas). Cuando resultan ser malignos suelen ser carcinomas broncogénicos que se consideran en estadio I de la clasificación TNM, siendo potencialmente curables mediante la resección. Entre el 10 y el $20 \%$ de los NPS malignos son metastásicos de origen extrapulmonar. El propósito primario de la caracterización de los nódulos pulmonares solitarios es determinar la verosimilitud de la malignidad de tales nódulos con el objetivo de planificar el tratamiento. El método radiológico diagnóstico estándar utilizado ha sido el TAC y, aunque se han empleado modalidades nuevas de TAC como la que utiliza contraste yodado o bien el TAC con densitometría de alta resolución (HRCT), no se ha conseguido evitar las limitaciones diagnósticas de esta téc-

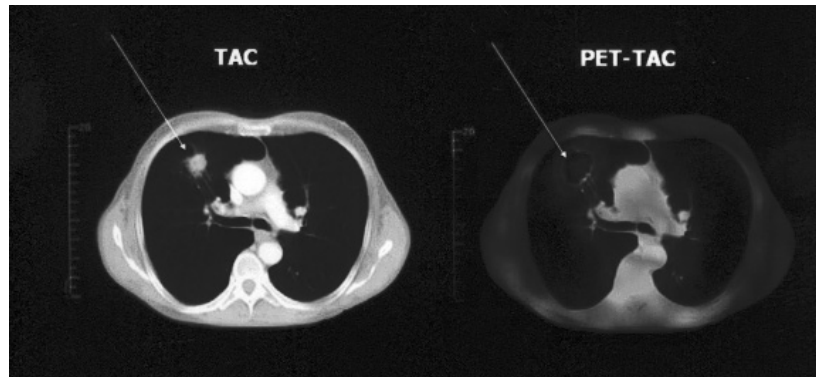

Fig. 3. Caracterización de un nódulo pulmonar solitario. La elevada captación de FDG indica malignidad.

nica (clasifica como indeterminados muchos NPS). Lo mismo ha ocurrido empleando la RM. La sensibilidad de la PET-FDG en esta situación clínica es del 93 al $100 \%$ y la especificidad del 78 al $88 \%$, con una exactitud diagnóstica del 92 al 94\%. La PET ha demostrado tener gran capacidad para diferenciar entre los de carácter benigno y maligno (Fig. 3). Estos valores mejoran cuando no se incluyen pacientes con procesos infecciosos-inflamatorios activos como tuberculosis o granulomas, cuando el tamaño del NPS es $>1,5 \mathrm{~cm}, y$ también puede mejorar la diferenciación benigno/ maligno si se realiza estudio semicuantitativo calculando el SUV (standard uptake value o valor estándar de captación). Cuando un NPS se informa como maligno en una PET, la probabilidad de malignidad es muy alta y cuando la PET es normal, la probabilidad de que un NPS sea maligno es muy baja además de tener un gran valor predictivo negativo. En diferentes estudios se ha descrito la influencia de la PET en el manejo terapéutico de los pacientes, en unos casos al evitar otros procedimientos diagnósticos invasivos, y en otros por evitar cirugías al detectar metástasis ganglionares y/o a distancia no sospechadas previamente ${ }^{36-40}$.

Actualmente, los criterios de indicación de la PET en NPS son ${ }^{1,2}$ :

- Pacientes con NPS $<4 \mathrm{~cm}$, radiológicamente indeterminado por TAC. Se exigirá exploración física y analítica completas.

- No se realizará PET como prueba de cribado poblacional ni en pacientes con diagnóstico previo de cáncer reciente.

\section{Cáncer colorrectal}

En el adenocarcinoma colorrectal son comunes las metástasis y la recurrencia tras resección quirúrgica con intención curativa del tumor primario. La utilidad clínica de la PET-FDG consiste en su relativa capacidad de predecir si la intervención quirúrgica será efectiva en el tratamiento del paciente. La sospecha de di- 


\section{J. P. Suárez Fernández y cols.}

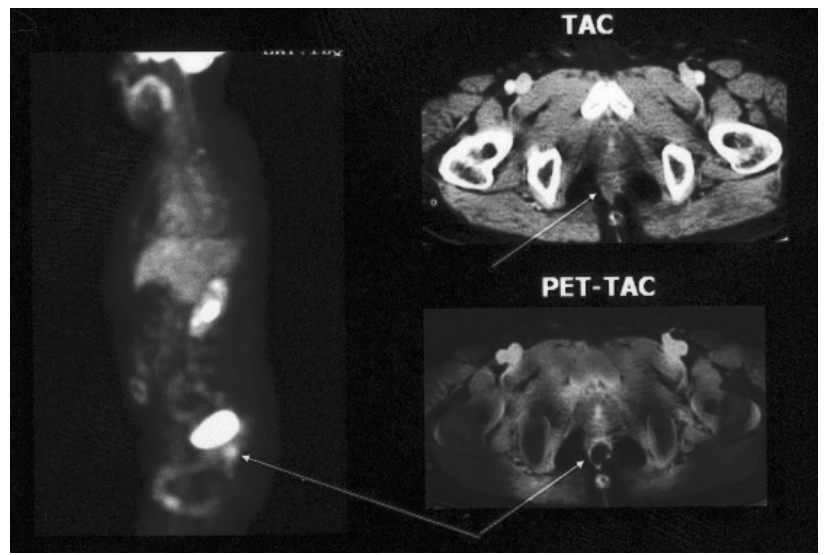

Fig. 4. Recidiva presacra en cáncer colorrectal, con imagen dudosa por TAC.

cha recurrencia puede estar basada en datos clínicos, de imagen o por elevación de marcadores y la PETFDG podría ayudar a contrastar esa sospecha. Por otro lado, la detección de las metástasis extrahepáticas es importante ya que evitaría cirugía innecesaria. La mayoría de los trabajos analizan la capacidad de la PET-FDG en la re-estadificación tumoral (Fig. 4), cuando después de haber sido intervenido el paciente del tumor primario colorrectal se presenta sospecha de recurrencia local o a distancia, fundamentalmente a nivel hepático, que es el lugar de más frecuente diseminación. Tanto para la estadificación en general como para el diagnóstico de recurrencia locorregional, de metástasis hepáticas y de otras metástasis a distancia, la PET-FDG presenta valores diagnósticos superiores a los demás medios de diagnóstico convencionales, y en concreto frente a la TAC, que es la técnica más habitual. Los resultados de la PET-FDG conducen a un cambio en la actitud terapéutica en un alto porcentaje de pacientes (22-68\%), en unos casos evitando la cirugía cuando la PET detecta enfermedad irresecable (18$50 \%$ casos); por ejemplo, al detectar más lesiones hepáticas en casos en los que se suponía una única, o al detectar lesiones a distancia no sospechadas modificando la estadificación tumoral, o, por el contrario, al indicar una cirugía con intención curativa $110-28 \%$ casos). Las cirugías innecesarias evitadas por la PET-FDG podrían dar lugar a una reducción en los costes de tratamiento, además de reducir la morbilidad asociada y mejorar la calidad de vida de los pacientes. La PET-FDG puede valorar la respuesta del cáncer rectal al tratamiento neoadyuvante: permite detectar dicha respuesta en el $100 \%$ de los casos frente al $78 \%$ de la TAC, según recientes estudios. Igualmente, se objetiva una mayor supervivencia libre de enfermedad en los

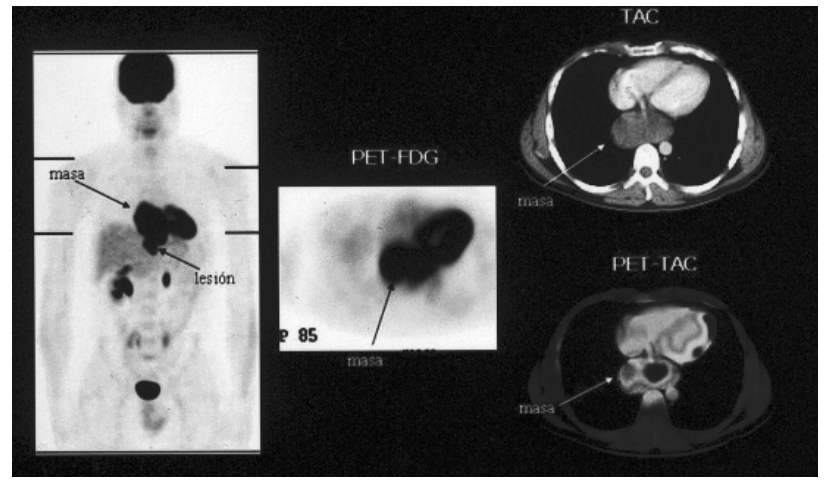

Fig. 5. Linfoma no-Hodgkin tras tratamiento quimioterápico. Masa mediastínica y lesión en abdomen superior de alto grado de malignidad.

pacientes estadificados mediante PET, lo que se relacionó con una mejor selección de pacientes que se beneficiarán de la cirugía. En otros casos, la PET-FDG permite guiar la biopsia disminuyendo el número de biopsias negativas 41-55. $^{2}$.

Actualmente, los criterios de indicación de la PET en cáncer colorrectal son ${ }^{1,2}$ :

- Pacientes con sospecha clínica, radiológica o analítica de recurrencia tras la realización de todas las pruebas diagnósticas convencionales, susceptibles de cirugía radical.

- No se realizará PET con fines de estadificación o valoración del tumor primario, ni en caso de ausencia de elevación de marcadores tumorales o en caso de no haberse realizado colonoscopia o TAC, si procede.

\section{Linfomas}

La efectividad de la PET-FDG en esta patología consiste en su capacidad de determinar la malignidad o benignidad de las lesiones encontradas y si el tratamiento aplicado va siendo eficaz para el paciente, por lo cual la PET-FDG (Fig. 5) se utiliza también en la reestadificación de estos enfermos tras los ciclos de tratamiento o quimioterapia. Existen dos indicaciones clínicas en las que la PET ha demostrado gran capacidad diagnóstica:

1) Valoración de tejido viable tumoral en masa residual detectada por TAC tras tratamiento, con sensibilidad comparable o superior a la TAC, pero con una especificidad muy superior a ésta, con significación estadística, permitiendo valorar con gran exactitud la presencia de tejido viable donde la TAC presenta resultados equívocos. En este sentido, la PET-FDG juega un papel en el manejo terapéutico de pacientes con linfomas al identificar aquellos que requieren tratamiento adicional. Se ha comprobado una superviven- 
cia libre de enfermedad significativamente superior en pacientes con PET-FDG negativas, lo que implica que la PET-FDG tiene mayor valor tanto diagnóstico como pronóstico que la TAC.

2) En la estadificación inicial y re-estadificación, para valorar afectación ganglionar y extraganglionar e infiltración de médula ósea, sus parámetros son superiores a la TAC, siendo especialmente útil en los linfomas de alto grado de agresividad. La PET-FDG permite detectar las mismas lesiones que la TAC pero, además, detecta otras que no habían sido sospechadas con otras técnicas diagnósticas (en un 16-48\% de pacientes), lo que conduce a un cambio en la estadificación que se traduce, en muchos casos, en cambios en la estrategia terapéutica en un número considerable de pacientes. Al tener similares resultados que la biopsia de médula ósea, la PET-FDG podría utilizarse en sustitución de esta técnica invasiva, que, por otro lado, presenta en algunos casos falsos negativos. En otros casos la PET-FDG serviría de guía para indicar el lugar más favorable a biopsiar y reducir el número de biopsias negativas s-68 $^{5-6}$.

Actualmente, los criterios de indicación de la PET en los linfomas son 1,2 :

- Pacientes con evidencia histológica de linfoma maligno (Hodgkin y no Hodgkin), en fase de estadificación, tras realizar pruebas convencionales.

- Pacientes con masa residual tras terapia de linfoma maligno.

\section{Tumores primarios de origen desconocido}

En ocasiones, el diagnóstico de cáncer parte del hallazgo de marcadores tumorales séricos elevados o por detección de metástasis regionales o a distancia, siendo los métodos diagnósticos convencionales no concluyentes. Es evidente que los marcadores tumorales cuando están elevados indican con más especificidad que la PET-FDG que existe un tumor activo, aunque estos marcadores por sí solos no objetivan la localización del tumor. Sin embargo, siendo la PET-FDG más sensible, puede determinar la localización y contribuir a diagnosticar la naturaleza y extensión (estadificación) del tumor. En efecto, la PET-FDG puede, en diferentes neoplasias gastrointestinales (hígado y tumores de colon y recto) y de otras localizaciones, detectar recurrencias y tumores primarios o metastásicos en casos en que existe elevación del marcador CEA, o bien cuando los datos de la TAC y RM son equívocos, con unos datos de sensibilidad y exactitud diagnóstica altas $^{69-77}$.

Actualmente, los criterios de indicación de la PET en el cáncer de origen desconocido son ${ }^{1,2}$ :
- Pacientes con metástasis de origen desconocido sin evidencia de tumor primario con las técnicas diagnósticas convencionales y que sean susceptibles de tratamiento radical.

- No se realizará PET en pacientes con metástasis generalizadas.

\section{Melanoma}

La incidencia del melanoma maligno está aumentando con más rapidez que otras patologías tumorales malignas. Los melanomas pueden metastatizar en cualquier parte del cuerpo, siendo muy difícil predecir la localización de estas metástasis. A causa de la pobre respuesta a la inmunoterapia y quimioterapia es importante detectarlas con rapidez y extirparlas, ya que el pronóstico de un enfermo con metástasis distante es de 4 a 6 meses de vida.

Para la estadificación de los melanomas se utilizan diversas técnicas como radiografía de tórax, ultrasonidos, TAC, RM, gammagrafía, biopsias, etc. La ventaja que ofrece la PET-FDG se basa en que, con un solo examen, se tiene una visión del cuerpo entero (Fig. 6). Ello puede suministrar información importante sobre la malignidad del proceso patológico. Por lo anterior, se está utilizando la PET-FDG para la estadificación de los melanomas siempre que las lesiones sean mayores de $5 \mathrm{~mm}$. La PET-FDG puede ser útil en la evaluación tumoral con una sensibilidad que se sitúa en un rango del 74 al $94 \%$, una especificidad que va del 67 al $93 \%$ y una exactitud diagnóstica del $92 \%$. Comparada la PET-FDG con el TAC, la ultrasonografía y la gammagrafía planar, estas técnicas dan valores para la sensibilidad y especificidad inferiores a los de la PETFDG. Sin embargo, la incapacidad de la técnica en la detección de la enfermedad microscópica, hace necesario el complementar el estudio de la afectación gan-

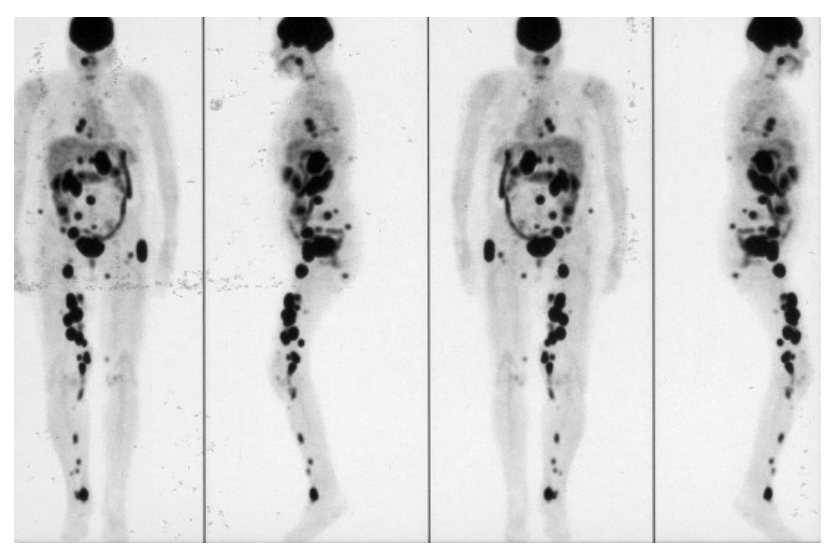

Fig. 6. Melanoma maligno: diseminación tumoral ósea, ganglionar, pulmonar y de partes blandas. 


\section{J. P. Suárez Fernández y cols.}

glionar con la técnica del ganglio centinela. Como elemento a destacar, la utilidad clínica de esta técnica consiste en su capacidad de determinar si existe afectación ganglionar y si la intervención quirúrgica sería efectiva en este contexto, $y$ en el tratamiento del paciente con sospecha de recurrencia operable ${ }^{78-80}$.

Actualmente, los criterios de indicación de la PETFDG en melanoma son ${ }^{1,2}$ :

- Pacientes con sospecha de recurrencia, susceptibles de cirugía radical, tras la realización de todas las pruebas diagnósticas convencionales.

- Sólo se realizará PET como estudio de estadificación en caso de melanomas con alta probabilidad de metastatización (Breslow $>4 \mathrm{~mm}$ ).

\author{
Correspondencia: \\ Dr. A. Maldonado Suárez \\ Centro PET Complutense \\ Bartolomé Cossío, 10 \\ E-28040 Madrid \\ E-mail: director@petmadrid.com \\ Web: www.petmadrid.com
}

\section{Bibliografía}

1. Tomografía por emisión de positrones (PET) con 18FDG en oncología clínica (Revisión Sistemática). Informe de la Agencia de Evaluación de Tecnologías Sanitarias № 30. Instituto de Salud Carlos III. Ministerio de Sanidad y Consumo. Madrid, Noviembre de 2001.

2. Protocolo de uso tutelado para la recogida de información sobre la utilización de la 18FDG-PET. Informe de la Agencia de Evaluación de Tecnologías Sanitarias. Instituto de Salud Carlos III. Ministerio de Sanidad y Consumo. Madrid, Mayo de 2002.

3. Henze M, Mohammed A, Schlemmer HP, Herfarth KK, Hoffner S, Haufe S, et al. PET and SPECT for Detection of Tumor Progression in Irradiated Low-Grade Astrocytoma: A Receiver-Operating-Characteristic Analysis. J Nucl Med 2004; 45:579-86.

4. Jacobs AH, Dittmar C, Winkeler A, Garlip G, Heiss WD. Molecular imaging of gliomas. Mol Imaging 2002; 1:309-35.

5. Lee JK, Liu RS, Shiang HR, Pan DH. Usefulness of Semiquantitative FDG-PET in the Prediction of Brain Tumor Treatment Response to Gamma Knife Radiosurgery. J Comput Assist Tomogr 2003; 27:525-9.

6. Ricci PE, Karis JP, Heiserman JE, Fram EK, Bice AN, Drayer BP. Differentiating recurrent tumor from radiation necrosis: Time for re-evaluation of positron emission tomography? Am J Neuroradiol 1998; 19: 407-13.
7. Chao ST, Suh JH, Raja S, Lee SY, Barnett G. The sensitivity and specificity of FDG PET in distinguishing recurrent brain tumor from radionecrosis in patients treated with stereotactic radiosurgery. Int J Cancer 2001;96:191-7.

8. Gibson MK, Forastiere AA. Multidisciplinary approaches in the management of advanced head and neck tumors: state of the art. Curr Opin Oncol 2004; 16:220-4.

9. Douglas WG, Rigual NR, Loree TR, Wiseman SM, AlRawi S, Hicks WL Jr. Current concepts in the management of a second malignancy of the lung in patients with head and neck cancer. Curr Opin Otolaryngol Head Neck Surg 2003; 11:85-8.

10. Aigner RM, Schultes G, Wolf G, Yamashita Y, Sorantin E, Karcher H. 18F-FDG PET: early postoperative period of oro-maxillo-facial flaps. Nuklearmedizin 2003; 42:210-4

11. Di Martino E, Nowak B, Krombach GA, Sellhaus B, Hausmann R, Cremerius U, et al. Results of pretherapeutic lymph node diagnosis in head and neck tumors. Clinical value of 18-FDG positron emission tomography (PET). Laryngorhinootologie 2000; 79:201-6.

12. Kresnik E, Mikosch P, Gallowitsch HJ, Kogler D, Wieser $S$, Heinisch $M$ et al. Evaluation of head and neck cancer with 18F-FDG PET: a comparison with conventional methods. Eur J Nucl Med 2001; 28:816-21.

13. Kao CH, ChangLai SP, Chieng PU, Yen RF, Yen TC. Detection of recurrent or persistent nasopharyngeal carcinomas after radiotherapy with 18-fluoro-2-deoxyglucose positron emission tomography and comparison with computed tomography. J Clin Oncol 1998; 16:3550-5.

14. Adams S, Baum RP, Stuckensen T, Bitter K, Hor G. Prospective comparison of 18F-FDG PET with conventional imaging modalities (CT, MRI, US) in lymph node staging of head and neck cancer. Eur J Nucl Med 1998; 25:1255-60.

15. Goerres GW, Haenggeli CA, Allaoua M, Albrecht SR, Dulguerov $P, B e c k e r M$, et al. Direct comparison of F-18FDG PET and ultrasound in the follow-up of patients with squamous cell cancer of the head and neck. Nuklearmedizin 2000; 39:246-50.

16. Slevin NJ, Collins CD, Hastings DL, Waller ML, Johnson RJ, Cowan RA, et al. The diagnostic value of positron emission tomography (PET) with radiolabelled fluorodeoxyglucose (18F-FDG) in head and neck cancer. J Laryngol Otol 1999; 113:548-54.

17. Nowak B, Di Martino E, Janicke S, Cremerius U, Adam $G$, Zimny $M$, et al. Diagnostic evaluation of malignant head and neck cancer by F-18-FDG PET compared to CT/MRI. Nuklearmedizin 1999; 38:312-8.

18. Masahiro Iwata, Kanji Kasagi, Takashi Misaki, Keiichi Matsumoto, Yasuhiro lida, Takayoshi Ishimori, et al. Comparison of whole-body 18F-FDG PET, 99mTc-MIBI SPET, and post-therapeutic 1311-Na scintigraphy in the detection of metastatic thyroid cancer. Eur J Nucl Med Mol Imaging 2004; 31:491-8. 
19. Schluter B, Bohuslavizki KH, Beyer W, Plotkin M, Buchert $\mathrm{R}$, Clausen M. Impact of FDG PET on patients with differentiated thyroid cancer who present with elevated thyroglobulin and negative 1311 scan. J Nucl Med 2001; 42:71-6.

20. Yeo JS, Chung JK, So Y, Kim S, Lee E, Lee DS, et al. F18-fluorodeoxyglucose positron emission tomography as a presurgical evaluation modality for I-131 scannegative thyroid carcinoma patients with local recurrence in cervical lymph nodes. Head Neck 2001; 23:94-103.

21. Grunwald F, Kalicke T, Feine U, Lietzenmayer R, Scheidhaver K, Dietlein $M$, et al. Fluorine-18 fluorodeoxyglucose positron emission tomography in thyroid cancer: results of a multicentre study. Eur J Nucl Med 1999; 26:1547-52.

22. Conti PS, Durski JM, Bacqai F, Grafton ST, Singer PA Imaging of locally recurrent and metastatic thyroid cancer with positron emission tomography. Thyroid 1999; 9:797-804.

23. Wang W, Macapinlac $H$, Larson SM, Yeh SD, Akhurst T, Finn RD, et al. [18F]-2-fluoro-2- deoxy-D-glucose positron emission tomography localizes residual thyroid cancer in patients with negative diagnostic (131)l whole body scans and elevated serum thyroglobulin levels. J Clin Endocrinol Metab 1999; 84:2291-302.

24. Frilling A, Gorges R, Tecklenborg K, Gassmann P, Bockhorn $M$, Clausen $M$, et al. Value of preoperative diagnostic modalities in patients with recurrent thyroid carcinoma. Surgery 2000; 128:1067-74.

25. Kelly RF, Tran T, Holmstrom A, Murar J, Segurola RJ Jr. Accuracy and Cost-Effectiveness of [18F]-2-Fluoro-Deoxy-D-Glucose-Positron Emission Tomography Scan in Potentially Resectable Non-small Cell Lung Cancer. Chest 2004; 125:1413-23.

26. Halter G, Buck AK, Schirrmeister H, Wurziger I, Liewald F, Glatting G, et al. [F] 3-deoxy-3'-fluorothymidine positron emission tomography: Alternative or diagnostic adjunct to 2-[f]-fluoro-2-deoxy-d-glucose positron emission tomography in the workup of suspicious central focal lesions? J Thorac Cardiovasc Surg 2004; 127:1093-9.

27. Blum R, MacManus MP, Rischin D, Michael M, Ball D, Hicks RJ. Impact of positron emission tomography on the management of patients with small-cell lung cancer: preliminary experience. Am J Clin Oncol 2004; 27: 16471.

28. ChangLai SP, Tsai SC, Chou MC, Ho YJ, Kao CH. Whole body 18F-2-deoxyglucose positron emission tomography to restage non-small cell lung cancer. Oncol Rep $2001 ; 8: 337-9$

29. Kutlu CA, Pastorino U, Maisey M, Goldstraw P. Selective use of PET scan in the preoperative staging of NSCLC. Lung Cancer 1998; 21:177-84.

30. Tatsumi M,Yutani K, Nishimura T. Evaluation of lung cancer by $99 \mathrm{mTc}$-tetrofosmin SPECT: comparison with [18F]FDG-PET. J Comput Assist Tomog 2000; 24:57480.
31. Marom EM, McAdams HP, Erasmus JJ, Goodman PC, Culhane DK, Coleman RE, et al. Staging non-small cell lung cancer with whole-body PET. Radiology 1999; 212:803-9.

32. Weder W, Schmid RA, Bruchhaus H, Hillinger S, von Schulthess GK, Steinert HC. Detection of extrathoracic metastases by positron emission tomography in lung cancer. Ann Thorac Surg 1998; 66:886-92.

33. Vansteenkiste JF, Stroobants SG, Dupont PJ, De Leyn PR, De Wever WF, Verbeken EK, et al. FDG-PET scan in potentially operable non-small cell lung cancer: do anatometabolic PET-CT fusion images improve the localisation of regional lymph node metastases? The Leuven Lung Cancer Group. Eur J Nucl Med 1998; 25:1495-501.

34. Vansteenkiste JF, Stroobants SG, De Leyn PR, Dupont PJ, Verbeken EK. Potential use of FDG-PET scan after induction chemotherapy in surgically staged Illa-N2 nonsmall-cell lung cancer: a prospective pilot study. The Leuven Lung Cancer Group. Ann Oncol 1998; 9:1193-8.

35. Bury T, Barreto A, Daenen F, Barthelemy N, Ghaye B, Rigo P. Fluorine-18 deoxyglucose positron emission tomography for the detection of bone metastases in patients with non-small cell lung cancer. Eur J Nucl Med 1998; 25:1244-7.

36. Fischer BM, Mortensen J, Dirksen A, Eigtved A, Hoigaard L. Positron emission tomography of incidentally detected small pulmonary nodules. Nucl Med Commun 2004; 25:3-9.

37. Praver HW, Weber WA, Romer W, Treumann T, Ziegler $\mathrm{SI}$, Schwaiger M. Controlled prospective study of positron emission tomography using the glucose analogue [18f]fluorodeoxyglucose in the evaluation of pulmonary nodules. Br J Surg 1998; 85:1506-11.

38. Lowe VJ, Fletcher JW, Gobar L, Lawson M, Kirchner P, Valk $P$, et al. Prospective investigation of positron emission tomography in lung nodules. J Clin Oncol 1998; 16:1075-84.

39. Hagberg RC, Segall GM, Stark P, Burdon TA, Pompili MF. Characterization of pulmonary nodules and mediastinal staging of bronchogenic carcinoma with F-18 fluorodeoxyglucose positron emission tomography. Eur J Cardiothorac Surg 1997; 12:92-7.

40. Dewan NA, Shehan CJ, Reeb SD, Gobar LS, Scott WJ, Ryschon K. Likelihood of malignancy in a solitary pulmonary nodule: comparison of Bayesian analysis and results of FDG-PET scan. Chest 1997; 112:416-22.

41. Ruers BS, Langenhoff N, Neeleman GJ, Jager S, Strijk Th, Wobbes F, et al. Value of Positron Emission Tomography With [F-18]Fluorodeoxyglucose in Patients With Colorectal Liver metastases: A Prospective Study T.J.M. J Clin Oncol 2002; 20:388-95.

42. Tzimas GN, Koumanis DJ, Meterissian S. Positron emission tomography and colorectal carcinoma: an update. J Am Coll Surg 2004; 198:645-52.

43. Schussler-Fiorenza CM, Mahvi DM, Niederhuber J, Rikkers LF, Weber SM. Clinical risk score correlates with 


\section{J. P. Suárez Fernández y cols.}

yield of PET scan in patients with colorectal hepatic metastases. J Gastrointest Surg 2004; 8:150-8.

44. Staib L, Schirrmeister H, Reske SN, et al. Is 18F-fluorodeoxyglucose positron emission tomography in recurrent colorectal cancer a contribution to surgical decision making? Am J Surg 2000; 180:1-5.

45. Boykin KN, Zibari GB, Lilien DL, McMillan RW, Aultman DF, McDonald JC. The use of FDG-positron emission tomography for the evaluation of colorectal metastases of the liver. Am Surg 1999; 65:1 183-5.

46. Ogunbiyi OA, Flanagan FL, Dehdashti F, Siegel BA, Trask DD, Birnbaum EH, et al. Detection of recurrent and metastatic colorectal cancer: comparison of positron emission tomography and computed tomography. Ann Surg Oncol 1997; 4:613-20.

47. Fong $Y$, Saldinger PF, Akhurst T, Macapinlac $H$, Yeung $\mathrm{H}$, Finn RD, et al. Utility of 18F-FDG positron emission tomography scanning on selection of patients for resection of hepatic colorectal metastases. Am J Surg 1999; 178:282-7.

48. Flanagan FL, Dehdashti F, Ogunbiyi OA, Kodner IJ, Siegel BA. Utility of FDG-PET for investigating unexplained plasma CEA elevation in patients with colorectal cancer. Ann. Surg 1998; 227:319-23.

49. Takeuchi O, Saito N, Koda K, Sarashina H, Nakajima $\mathrm{N}$. Clinical assessment of positron emission tomography for the diagnosis of local recurrence in colorectal cancer. Br J Surg 1999; 86:932-7.

50. Valk PE. Whole-body PET imaging with [18F]fluorodeoxyglucose in management of recurrent colorectal cancer. Arch Surg 1999; 134:503-11.

51. Flamen $P$, Stroobants $S$, Van Cutsem E, Dupont $P$, Bormans $G$, De Vadder N, et al. Additional value of wholebody positron emission tomography with fluorine-18-2fluoro-2-deoxy-D-glucose in recurrent colorectal cancer. J Clin Oncol 1999; 17:894-901.

52. Guillem JG, Puig-La CJJ, Akhurst T, Tickoo S, Ruo L, Minsky BD, et al. Prospective assessment of primary rectal cancer response to preoperative radiation and chemotherapy using 18-fluorodeoxyglucose positron emission tomography. Dis Colon Rectum 2000; 43:18-24.

53. Flamen $P$, Hoekstra OS, Homans F, Van Cutsem E, Maes A, Stroobants $S$, et al. Unexplained rising carcinoembryonic antigen (CEA) in the postoperative surveillance of colorectal cancer: the utility of positron emission tomography (PET). Eur J Cancer 2001; 37:862-9.

54. Strasberg SM, Dehdashti F, Siegel BA, Drebin JA, Linehan D. Survival of patients evaluated by FDGPET before hepatic resection for metastatic colorectal carcinoma: a prospective database study. Ann Surg 2001; 233:293-9.

55. Topal B, Flamen P, Aerts R, D'Hoore A, Filez L, Van Cutsem $E$, et al. Clinical value of whole-body emission tomography in potentially curable colorectal liver metastases. Eur J Surg Oncol 2001; 27:175-9.

56. Friedberg JW, Fischman A, Neuberg D, Kim H, Takvo- rian $\mathrm{T}, \mathrm{Ng}$ A., et al. FDG-PET is superior to gallium scintigraphy in staging and more sensitive in the follow-up of patients with de novo Hodgkin lymphoma: a blinded comparison. Leuk Lymphoma 2004; 45:85-92.

57. Filmont JE, Yap CS, Ko F, Vranjesevic D, Quon A, Margolis DJ, et al. Conventional imaging and 2-deoxy-2[(18)F]fluoro-D-glucose positron emission tomography for predicting the clinical outcome of patients with previously treated Hodgkin's disease. Mol Imaging Biol 2004; 6:47-54.

58. Naumann R, Beuthien-Baumann B, Reiss A, Schulze J, Hanel A, Bredow J, et al. Substantial impact of FDG PET imaging on the therapy decision in patients with earlystage Hodgkin's lymphoma. Br J Cancer 2004; 90: 6205.

59. Jerusalem G, Beguin Y, Fassotte MF, Najiar F, Paulus P, Rigo $P$, et al. Whole-body positron emission tomography using 18F-fluorodeoxyglucose for posttreatment evaluation in Hodgkin's disease and non-Hodgkin's lymphoma has higher diagnostic and prognostic value than classical computed tomography scan imaging. Blood 1999; 94:429-33.

60. Stumpe KD, Urbinelli M, Steinert HC, Glanzmann C, Buck A, von Schulthes $G$. Whole-body positron emission tomography using fluorodeoxyglucose for staging of lymphoma: effectiveness and comparison with computed tomography. Eur J Nucl Med 1998; 25:721-8.

61. Hoh CK, Glaspy J, Rosen P, Dahlbom M, Lee SJ, Kunkel $L$, et al. Wholebody FDG-PET imaging for staging of Hodgkin's disease and lymphoma. J Nucl Med 1997; 38:343-8

62. Cremerius U, Fabry U, Kroll U, Zimny M, Neuerburg J, Osieka R, et al. Clinical value of FDG PET for therapy monitoring of malignant lymphoma-results of a retrospective study in 72 patients. Nuklearmedizin 1999; 38:24-30

63. Carr R, Barrington SF, Madan B, O'Doherty MJ, Saunders CA, van der Walt J, et al. Detection of lymphoma in bone marrow by whole-body positron emission tomography. Blood 1998; 91:3340-6.

64. Bangerter M, Moog F, Buchmann I, Kotzerke J, Griesshammer $M$, Hafner $M$, et al. Whole-body 2-[18F]-fluoro-2-deoxy-D-glucose positron emission tomography (FDG-PET) for accurate staging of Hodgkin's disease. Ann Oncol 1998; 9:1117-22.

65. Cremerius U, Fabry U, Neuerburg J, Zimny M, Osieka $\mathrm{R}$, Buell U. Positron emission tomography with 18F-FDG to detect residual disease after therapy for malignant lymphoma. Nucl Med Commun 1998; 19: 1055-63.

66. Spaepen K, Stroobants S, Dupont P, Van Steenweghen $S$, Thomas J, Vandenberghe $P$, et al. Prognostic value of positron emission tomography (PET) with fluorine-18 fluorodeoxyglucose ([18F]FDG) after first-line chemotherapy in non-Hodgkin's lymphoma: is [18F]FDG-PET a valid alternative to conventional diagnostic methods? J Clin Oncol 2001; 19: 414-9. 
67. Buchmann I, Reinhardt M, Elsner K, Bunjes D, Altehoefer C, Finke J, et al. 2-(fluorine-18)fluoro-2-deoxy-D-glucose positron emission tomography in the detection and staging of malignant lymphoma. A bicenter trial. Cancer 2001; 91:889-99.

68. Hueltenschmidt B, Sautter-Bihl ML, Lang O, Maul FD, Fischer J, Mergenthaler HG, et al. Whole body positron emission tomography in the treatment of Hodgkin disease. Cancer 2001; 91:302-10.

69. Jereczek-Fossa BA, Jassem J, Orecchia R. Cervical lymph node metastases of squamous cell carcinoma from an unknown primary. Cancer Treat Rev 2004; 30:153-64.

70. Alberini JL, Belhocine T, Hustinx R, Daenen F, Rigo P. Whole-body positron emission tomography using fluorodeoxyglucose in patients with metastases of unknown primary tumours (CUP syndrome). Nucl Med Commun 2003; 24:1081-6.

71. Delgado-Bolton RC, Fernandez-Perez C, Gonzalez-Mate A. Meta-analysis of the performance of 18F-FDG PET in primary tumor detection in unknown primary tumors. J Nucl Med 2003; 44:1301-14.

72. Assar OS, Fischbein NJ, Caputo GR, Kaplan MJ, Price DC, Singer MI, et al. Metastatic head and neck cancer: role and usefulness of FDG PET in locating occult primary tumors. Radiology 1999; 210:177-81.

73. Kole AC, Nieweg OE, Pruim J, Hoekstra HJ, Koops HS, Roodenburg JL, et al. Detection of unknown occult primary tumors using positron emission tomography. Cancer 1998; 82:1160-6.
74. Braams JW, Pruim J, Kole AC, Nikkels PG, Vaalburg W, Vermey $A$, et al. Detection of unknown primary head and neck tumors by positron emission tomography. Int J Oral Maxillofac Surg1997; 26:112-5.

75. Bohuslavizki KH, Klutmann S, Kroger S, Sonnemann U, Buchert R,Werner JA, et al. FDG PET detection of unknown primary tumors. J Nucl Med 2000; 41:816-22.

76. Gupta NC, Nicholson P, Bloomfield SM. FDG-PET in the staging work-up of patients with suspected intracranial metastatic tumors. Ann Surg 1999; 230:202-6.

77. Greven KM, Keyes JWJ,Williams DW, McGuirt WF, Joyce WT. Occult primary tumors of the head and neck: lack of benefit from positron emission tomography imaging with 2-[F-18]fluoro-2-deoxy-Dglucose. Cancer 1999; 86:114-8.

78. Fink AM, Holle-Robatsch S, Herzog N, Mirzaei S, Rappersberger $\mathrm{K}$, Lilgenau $\mathrm{N}$, et al. Positron emission tomography is not useful in detecting metastasis in the sentinel lymph node in patients with primary malignant melanoma stage I and II. Melanoma Res 2004; 14:141-5.

79. Gulec SA, Faries MB, Lee CC, Kirgan D, Glass C, Morton $\mathrm{DL}$, et al The role of fluorine-18 deoxyglucose positron emission tomography in the management of patients with metastatic melanoma: impact on surgical decision making. Clin Nucl Med 2003; 28:961-5.

80. Mijnhout GS, Hoekstra OS, van Tulder MW, Teule GJ, Deville WL. Systematic review of the diagnostic accuracy of (18)F-fluorodeoxyglucose positron emission tomography in melanoma patients. Cancer 2001; 91:153042. 